\title{
Clinical anatomy of the musculoskeletal system in the hip region
}

\author{
Masahiro Tsutsumi ${ }^{1,2} \circledast$ Akimoto Nimura $^{3} \cdot$ Keiichi Akita $^{1}$
}

Received: 4 July 2021 / Accepted: 15 October 2021 / Published online: 22 October 2021

(c) The Author(s) 2021

\begin{abstract}
Although the hip joint is regarded as inherently stable, hip pain and injuries caused by traumatic/non-traumatic hip instability are relatively common in active individuals. A comprehensive understanding of hip anatomy may provide better insight into the relationships between hip stability and clinical problems. In this review, we present our recent findings on the hip morphological characteristics, especially focusing on the intramuscular tendon of the gluteus medius tendon and its insertion sites, hip capsular attachment on the anterosuperior region of the acetabular margin, and composition of the iliofemoral ligament. We further discussed the hip stabilization mechanism based on these findings. The characteristics of the gluteus medius tendon suggest that even a single muscle has multiple functional subunits within the muscle. In addition, the characteristics of the hip capsular attachment suggest that the width of the capsular attachment is wider than previously reported, and its wide area shows adaptive morphology to mechanical stress, such as bony impression and distribution of the fibrocartilage. The composition of the iliofemoral ligament and its relation to periarticular structures suggest that some ligaments should be defined based on the pericapsular structures, such as the joint capsule, tendon, and aponeurosis, and also have the ability to dynamically coordinate joint stability. These anatomical perspectives provide a better understanding of the hip stabilization mechanism, and a biomechanical study or an in vivo imaging study, considering these perspectives, is expected in the future.
\end{abstract}

Keywords Clinical anatomy $\cdot$ Gluteus medius $\cdot$ Hip joint capsule $\cdot$ Hip stability $\cdot$ Iliofemoral ligament

\section{Introduction}

Although the hip joint is one of the most inherently stable joints (Neumann 2020), hip pain and injuries, caused by hip instability are problematic and common in active individuals (Kalisvaart and Safran 2015; Kemp et al. 2020; Mosler et al. 2015; Safran 2019). The management of hip pain and injuries has recently evolved with remarkable advances in imaging modalities and arthroscopic techniques (Glick et al. 2014; Lynch et al. 2013). Since clinicians have to fully interpret the information gained from new technologies,

Masahiro Tsutsumi

tsutsumi.fana@tmd.ac.jp

1 Department of Clinical Anatomy, Graduate School of Medical and Dental Sciences, Tokyo Medical and Dental University, 1-5-45 Yushima, Bunkyo-ku, Tokyo 113-8519, Japan

2 Inclusive Medical Science Research Institute, Morinomiya University of Medical Sciences, Osaka, Japan

3 Department of Functional Joint Anatomy, Graduate School of Medical and Dental Sciences, Tokyo Medical and Dental University, Tokyo, Japan a comprehensive understanding of the hip anatomy may help its interpretation and promote further evolution in hip management.

We investigated the musculoskeletal system in the hip region while focusing on the relationships between the intramuscular tendon and its bony attachment morphology, characteristics of the capsular attachment, and composition of the ligament. In this review, we present our research on the musculoskeletal system in the hip region and discuss insights into the hip stabilization mechanism based on the findings.

\section{Functional subunit within the gluteus medius}

Clinical problems, such as a tear of the gluteus medius tendon, caused by its morphological characteristics, are a good example for reconsidering the hip stabilization mechanism of the muscle. The gluteus medius is the largest muscle among the hip abductor muscles and is vital for hip stability (Neumann 2010). Recently, tears of the gluteus medius 
tendon have been recognized as a potential cause of lateral hip pain, which is a relatively common clinical symptom (Pierce et al. 2018; Segal et al. 2007; Zhu et al. 2020). According to previous reports, tears of the anterior fibers of the gluteus medius tendon occur more frequently than those of the posterior fibers (Bunker et al. 1997; Connell et al. 2003; Davies et al. 2013). The relationships between the different frequencies of the tendinous tears within the muscle and the morphology of the gluteus medius may be important to facilitate early diagnosis with imaging, appropriate surgery, or rehabilitation protocols; however, few studies have focused on these relationships.

As originally described as "Wolff's law" (Wolff 1892), the bony morphology is adaptive to the muscular power loaded on it via the tendon and, some previous studies have also shown the example of the bony anatomical structure (Nozaki et al. 2015; Sato et al. 2018; Tamaki et al. 2014). Therefore, the bony morphology of the gluteus medius may be important in considering the relationship between the frequency of tears and morphology of the gluteus medius tendon. Moreover, the intramuscular tendon may also be vital to its relationships because intramuscular tendons with different characteristics, such as the length and thickness, might form a single myotendinous unit and its characteristics could reveal the accurate tendinous compositions and detail footprints (Arai et al. 2008; Mochizuki et al. 2008; Nimura et al. 2014; Sato et al. 2012, 2018).

Regarding the bony morphology, the gluteus medius mainly originates from the outer surface of the ilium, which is composed of two parts, the posterior and anterolateral parts, based on the directions of the surface of the ilium (Fig. 1A). Based on this bony morphology, the posterior and anterolateral parts of the intramuscular tendons can be roughly distinguished (Fig. 1B). The posterior part is long and thick and converges on the superoposterior facet of the greater trochanter, which can be identified using microcomputed tomography (micro-CT) (Fig. 1C). The anterolateral part is short and thin and runs posteroinferiorly toward the lateral facet of the greater trochanter, also identifiable by micro-CT. Therefore, the gluteus medius is regarded as having two subunits within the muscle (posterior and anterolateral parts) based on the bony morphology and intramuscular tendons (Tsutsumi et al. 2019a).

Concerning the frequency of tendinous tears within the muscle, the thin anterolateral part may be more prone to tears than the thick posterior part. This morphological suggestion corresponds to the clinical frequency of tears (Bunker et al. 1997; Connell et al. 2003; Davies et al. 2013). In addition, the convergence and thickness of the posterior part may be vital in hip stability, as indicated by electromyographic studies (Gottschalk et al. 1989; Semciw et al. 2013). Based on the corresponding relationships between the morphology and function of the subunits within the gluteus medius, the perspective that even a single muscle has multiple functional subunits within the muscle may provide better insights into the hip stabilization mechanism.

\section{Capsular attachment on the anterosuperior acetabular margin}

Capsular attachment of the hip joint on the acetabular margin is illustrated as linear in an anatomical textbook (Gray 1985). On the other hand, the iliofemoral ligament
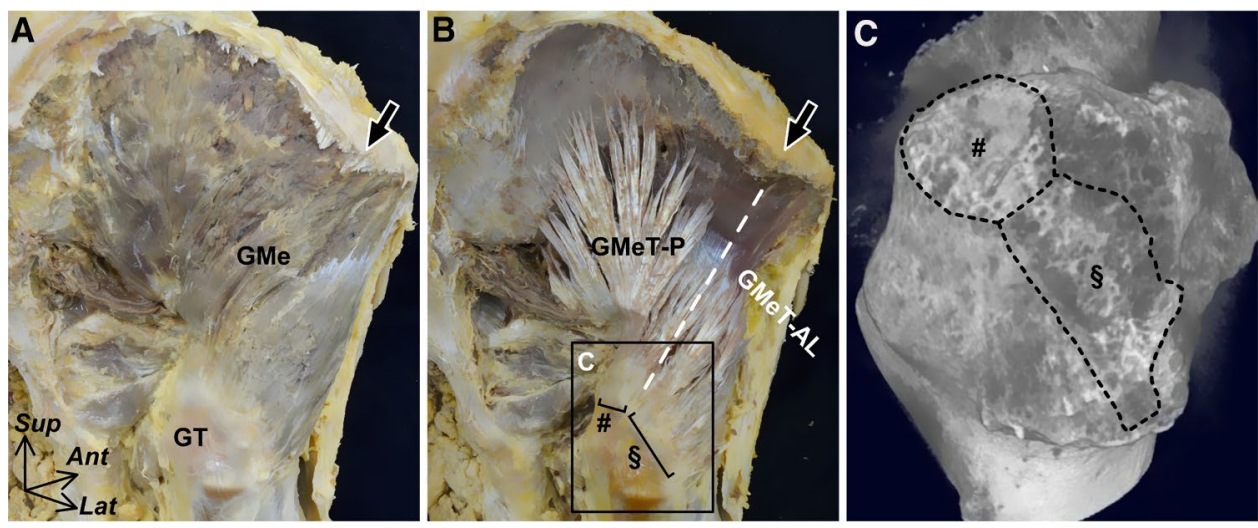

Fig. 1 Intramuscular tendon of the gluteus medius and its insertion sites. Posterolateral aspects of the right hip. A Gluteus medius (GMe) mainly originates from the outer surface of the ilium, and the arrow indicates the inflection point on the iliac crest between the posterior and anterolateral parts of the outer surface of the ilium. B After removal of the muscular portion of the GMe, the posterior and anterolateral parts of the intramuscular tendons (GMeT-P and GMeT-AL, respectively) can be identified based on this inflection point (arrow).
The number and section signs indicate the insertion of the GMeT-P and GMeT-AL on the greater trochanter (GT), respectively. C MicroCT image of the greater trochanter (GT) of the boxed region in B. Insertion of GMeT-P (number sign) and GMeT-AL (section sign) can be identified as the superolateral and lateral facets of the GT, respectively. Ant anterior, Lat lateral and Sup superior. ( Modified from: Tsutsumi et al. 2019a) 
is regarded as the major stabilizer of the hip joint, and it proximally and widely attaches to the inferior area of the anterior inferior iliac spine (AIIS), which is located in the anterosuperior region of the acetabular margin (Neumann 2020). Regardless of the proximity of the acetabular margin, the relationships between the anterosuperior capsule and iliofemoral ligament are often ambiguously described. For example, the iliofemoral ligament is described as "an accessory band of" or being "formed on," "spreads triangularly on," or "reinforced" the anterosuperior capsule (Braus and Elze 1954; Gray 1858; Neumann 2020; Schafer and Thane 1894). Ambiguity regarding its relationships may be related to a lack of precise knowledge of capsular attachment in the anterosuperior region of the acetabular margin.

In other articular systems, the joint capsule is thought to have a thin structure. For example, in the shoulder joint, Clark and Harryman (1992) stated that the joint capsule, which is located in the deepest layer of the rotator cuff, is a thin continuous sheet of interwoven collagen fibrils, and its attachment to the humerus was also illustrated as linear in an anatomical textbook (Gray 1985). However, Nimura et al. (2012) concluded that the conventional anatomical study overestimated the attachment width of the rotator cuff, and the capsular attachment of the shoulder joint occupied a substantial area of the greater tuberosity by the precise discrimination between the actual cuff insertion and capsular attachment. In other articular systems apart from the shoulder joint, such as the elbow, wrist, thumb metacarpophalangeal joint, knee, and ankle, many anatomical studies have also shown that the capsular attachment is not always linear, and its width varies according to the bony location (Amaha et al. 2019; Nasu et al. 2018; Nimura et al. 2014; Saka et al. 2021; Sato et al. 2018; Shimura et al. 2016). Therefore, these anatomical studies suggest that the width of the capsular attachment is wider than previously reported in most articular systems.

Regarding the hip capsular attachment, the width also varies according to the location, and the attachment width on the inferior area of the AIIS is larger than that on the anterosuperior region of the acetabular margin (Fig. 2). Moreover, the capsular attachment on the inferior area of the AIIS corresponds to the bony impression, which is identified by micro-CT (Fig. 3A). This bony impression on the inferior area of the AIIS shows cortical bone thickening (Fig. 3B).

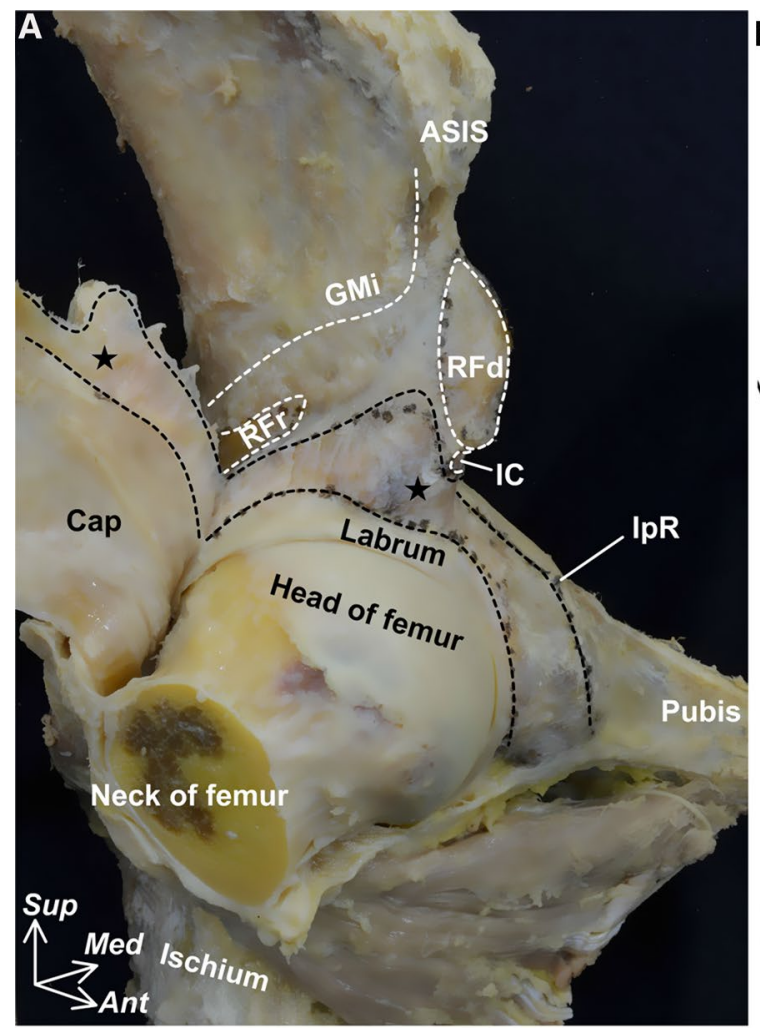

Fig. 2 Attachment width of hip joint capsule on the anterosuperior region of the acetabular margin. Anterolateral aspects of the right hip. A The joint capsule (Cap) is detached from the acetabular margin, and the black dashed lines indicate its osseous attachment. The white dashed lines indicate the origin sites of the gluteus minimus (GMi), iliocapsularis (Ic), and the direct and reflected head of the rectus

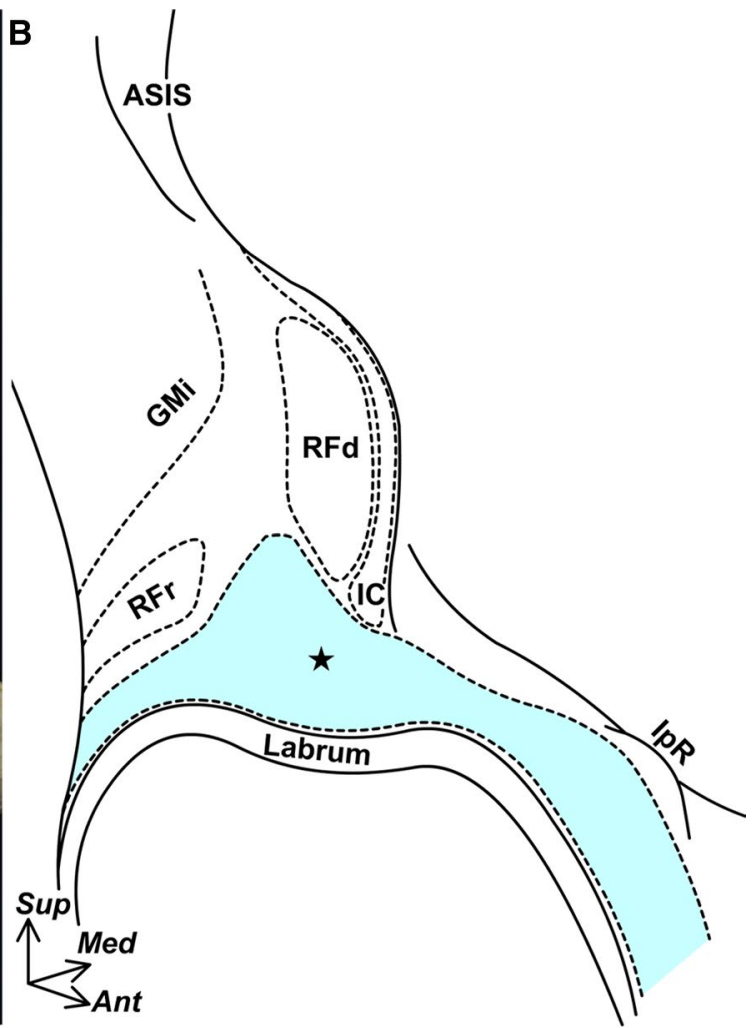

femoris (RFd and RFr, respectively). The star indicates the inferior area of the anterior inferior iliac spine. B Schematic illustration of the capsular attachment. ASIS anterior superior iliac spine, IpR iliopubic ramus, Labrum acetabular labrum, Ant anterior, Med medial, Sup superior. ( Modified from: Tsutsumi et al. 2019b) 


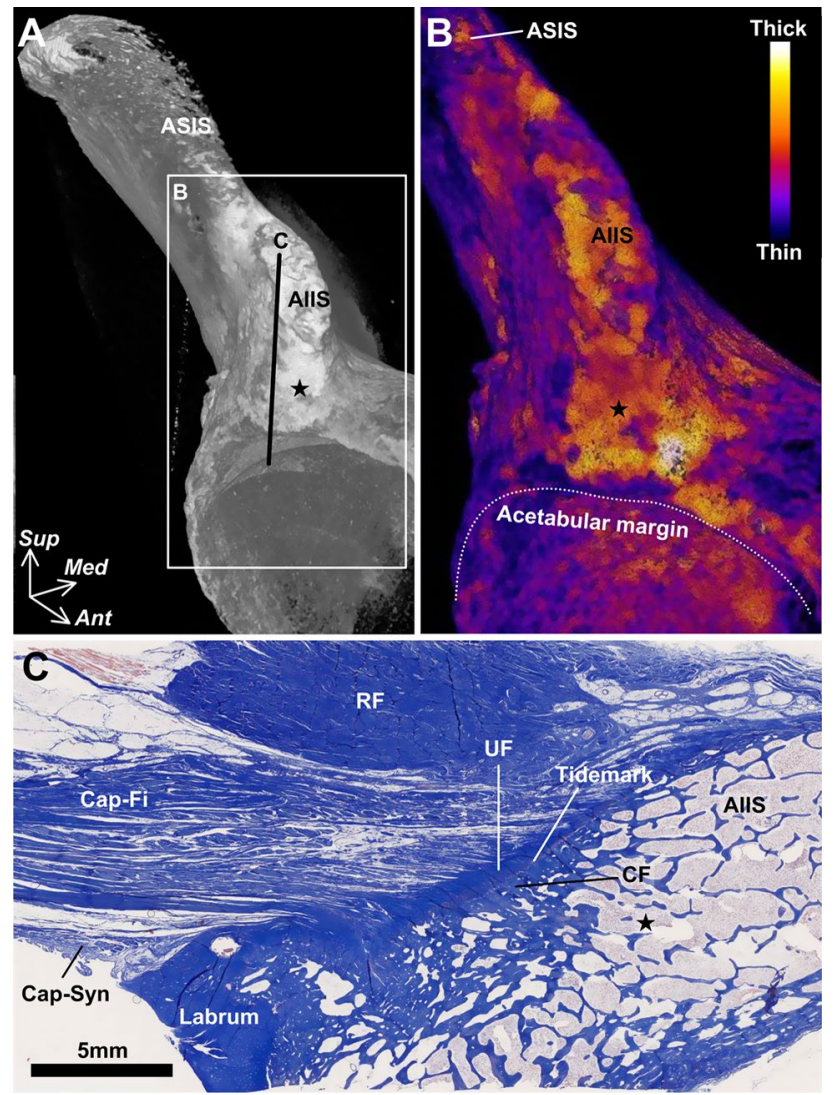

Fig. 3 Bone morphology and histological characteristics of the capsular attachment inferior to the anterior inferior iliac spine. A Microcomputed tomography image of the anterolateral aspect of the right hip. The inferior area (star) of the anterior inferior iliac spine (AIIS) has a bony impression. B Cortical thickening maps of the boxed region in A is visualized after image processing by Bone $\mathrm{J}$, which is ImageJ plug-in and can analyze the thickness of the structure of interest at a point (Hildebrand and Rüegsegger 1997; Doube et al. 2010). The brighter colors represent thicker cortical bone points. C Histological cross-section along line $\mathrm{C}$ in $\mathrm{A}$ is stained with Masson trichrome. The fibrous layer of the joint capsule (Cap-Fi) attaches to the inferior area of the AIIS via the fibrocartilage. ASIS anterior superior iliac spine, Cap-Syn synovial layer of the joint capsule, $C F$ calcified fibrocartilage, Labrum acetabular labrum, $R F$ rectus femoris, $U F$ uncalcified fibrocartilage, Ant anterior, Med medial, Sup superior. ( Modified from: Tsutsumi et al. 2019b)

These bony morphological features, particularly cortical bone thickening, are reported to correspond to the high tensile stress from dense connective tissues (Horiuchi et al. 2020; Tano et al. 2021). In addition, histological analysis revealed that the ligament-like structure independent of the joint capsule did not exist in the inferior area of the AIIS, and the joint capsule attaches to the inferior area of the AIIS via the fibrocartilage (Fig. 3C). As previously described, the distribution of fibrocartilage at the attachment site correlates highly with the levels of mechanical stress (Benjamin and Ralphs 1998). Therefore, the capsular attachment on the inferior area of the AIIS is highly adaptive to mechanical stress based on its attachment width, bony morphology, and histological features, and can be regarded as identical to the origin of the iliofemoral ligament (Tsutsumi et al. 2019b).

This anatomical knowledge of the hip capsular attachment highlights important clinical insights. Although hip instability after hip arthroscopy is generally considered a rare complication (Harris et al. 2013; Kowalczuk et al. 2013), its prevalence has increased in recent years (Ramos et al. 2017; Yeung et al. 2016). During some surgical procedures of hip arthroscopy, the joint capsule is partially detached from the inferior area of the AIIS for labral repair (Fry and Domb 2010; Krych et al. 2013) and AIIS decompression (Hapa et al. 2013; Michal et al. 2020), probably because its attachment is thought to be a linear structure. However, as mentioned above, the capsular attachment inferior to the AIIS, which is identical to the origin of the iliofemoral ligament, is highly adaptive to mechanical stress. Therefore, the detachment of the capsular attachment inferior to the AIIS during hip arthroscopy might have a great impact on hip instability, as also indicated by a biomechanical study (Fagotti et al. 2020), and may be related to an increase in the prevalence of hip instability after hip arthroscopy in recent years. Based on the morphological features in the area with wide capsular attachment, such as the bony impression and distribution of the fibrocartilage, as well as the hypothetical relationships between capsular detachment and hip instability, the perspective that the width of the capsular attachment is wider than previously reported is vital in considering joint stability.

\section{Dynamic stabilization mechanism via the iliofemoral ligament}

As described above, the capsular attachment on the inferior area of the AIIS is highly adaptive to mechanical stress and identical to the origin of the iliofemoral ligament (Tsutsumi et al. 2019b). Generally, the iliofemoral ligament spreads distally on the anterosuperior region of the hip joint, in an inverted Y, and is composed of the transverse and descending parts (Neumann 2020; Schafer and Thane 1894). The transverse part extends to the tubercle of the femur at the superolateral end of the intertrochanteric line and descending part to the inferomedial end of the line (Neumann 2020; Schafer and Thane 1894). Although the bony attachment of the iliofemoral ligament has been well investigated (Tamaki et al. 2020; Telleria et al. 2014; Wagner et al. 2012), the positional relationships between the iliofemoral ligament and the periarticular structures, such as the joint capsule or the hip muscles, have not been fully investigated.

Although the ligament is often assumed to be a bundlelike structure connecting bone to bone, drawing an arbitrary border between the ligament and periarticular structures is 
not appropriate in some cases. According to some histological textbooks, the ligament is classified as dense connective tissue, and its fibers are less regularly arranged than those of tendons, which are oriented parallel to the long axis, and more regularly than those of aponeuroses (Fawcett and Bloom 1986; Pawlina and Ross 2016). These fiber arrangements of the ligament are determined according to the local tensional demands, namely the relation to the surrounding anatomical structures, such as the joint capsule, tendon, or aponeurosis of the pericapsular muscles (Schleip 2012). For example, the humeroulnar joint capsule could not be separated from the tendinous septum between the flexor pronator muscles and these deep aponeuroses, and some of these complexes were the anterior bundle of the ulnar collateral ligament (Hoshika et al. 2019). In other articular systems apart from the elbow, such as knee and ankle, recent anatomical studies have also shown that the so-called "ligament" can be precisely defined based on pericapsular structures, such as the joint capsule, tendon, and aponeurosis (Amaha et al. 2019; Nasu et al. 2020).

Concerning the anterosuperior region of the hip joint, on which the iliofemoral ligament is considered to be located, the gluteus minimus and iliopsoas are located immediately superficial to the joint capsule (Fig. 4A). The gluteus minimus tendon is connected to the joint capsule (Fig. 4B and $\mathrm{C}$ ), and the lateral end of this connection is adjoined to the tubercle of the femur at the superolateral end of the intertrochanteric line (Fig. 4D). The deep aponeurosis of the iliopsoas is also connected to the joint capsule, and the inferomedial end of the anterior border corresponds to the inferomedial end of the intertrochanteric line. In addition, capsular thickening is identified in these connecting regions (Fig. 5). Therefore, the transverse and descending parts of the iliofemoral ligament could be regarded as joint capsules with fibers arranged according to their connection with the gluteus minimus tendon and deep aponeurosis of the iliopsoas, respectively (Tsutsumi et al. 2020).

This anatomical interpretation provides useful information regarding hip joint stability. In general, the iliofemoral ligament contributes to hip joint stability as the main static stabilizer (Myers et al. 2011; Walters et al. 2014). However, if the iliofemoral ligament is a joint capsule complex with a connection to the gluteus minimus tendon and deep aponeurosis of the iliopsoas, the iliofemoral ligament can dynamically coordinate the hip position during movement by transmitting the contraction force of the gluteus minimus and iliopsoas to the joint. Therefore, the iliofemoral ligament can be regarded as not only a static but also dynamic stabilizers. The precise definition of the ligament based on the pericapsular structures provides a new perspective that the so-called "ligament" has the ability to dynamically coordinate joint stability, which may provide better insights into the hip stabilization mechanism.
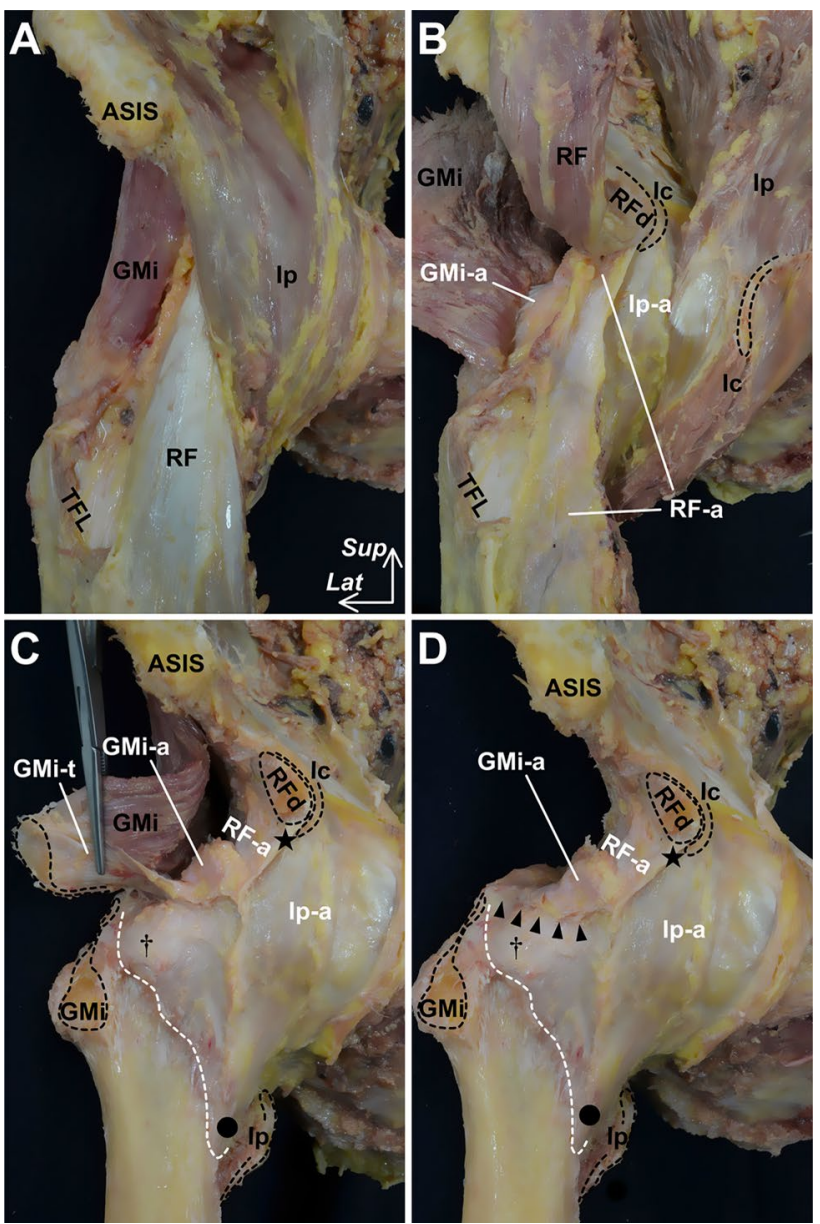

Fig. 4 Spatial geometry of the gluteus minimus, iliopsoas and hip joint capsule. Anterior aspects of the right hip. A Surface of the pericapsular muscles, including the gluteus minimus (GMi), iliopsoas (Ip), and rectus femoris (RF). B The muscles are reflected to identify their deep aponeuroses. C Some of the deep aponeuroses, which do not connect to the outer surface of the joint capsule, are removed. The deep aponeuroses of the GMi (GMi-a), Ip (Ip-a), and proximal aponeurosis of the rectus femoris (RF-a) are connected to the joint capsule. In addition, both the rectus femoris and iliopsoas are also removed because they do not attach to the joint capsule, except for the deep aponeuroses. After detaching the GMi insertion on the femur, the connection between the GMi tendon (GMi-t) and the joint capsule can be identified. D Arrowheads indicate the cut line of the connection between the GMi-t and the joint capsule. The lateral end of the connection is adjoined to the tubercle of the femur at the superolateral end of the intertrochanteric line (dagger). The inferomedial end of the anterior border of the Ip-a corresponds to the inferomedial end of the intertrochanteric line (circle). ASIS anterior superior iliac spine, Ic iliocapsularis, $R F d$ direct head of the RF, Star inferior area of the anterior inferior iliac, TFL tensor fasciae latae; white dashed line, distal margin of the joint capsule on the intertrochanteric line, Lat lateral and Sup superior. ( Modified from Tsutsumi et al. 2020)

\section{Conclusion}

This review highlights three anatomical perspectives. First, a single muscle has multiple functional subunits within 

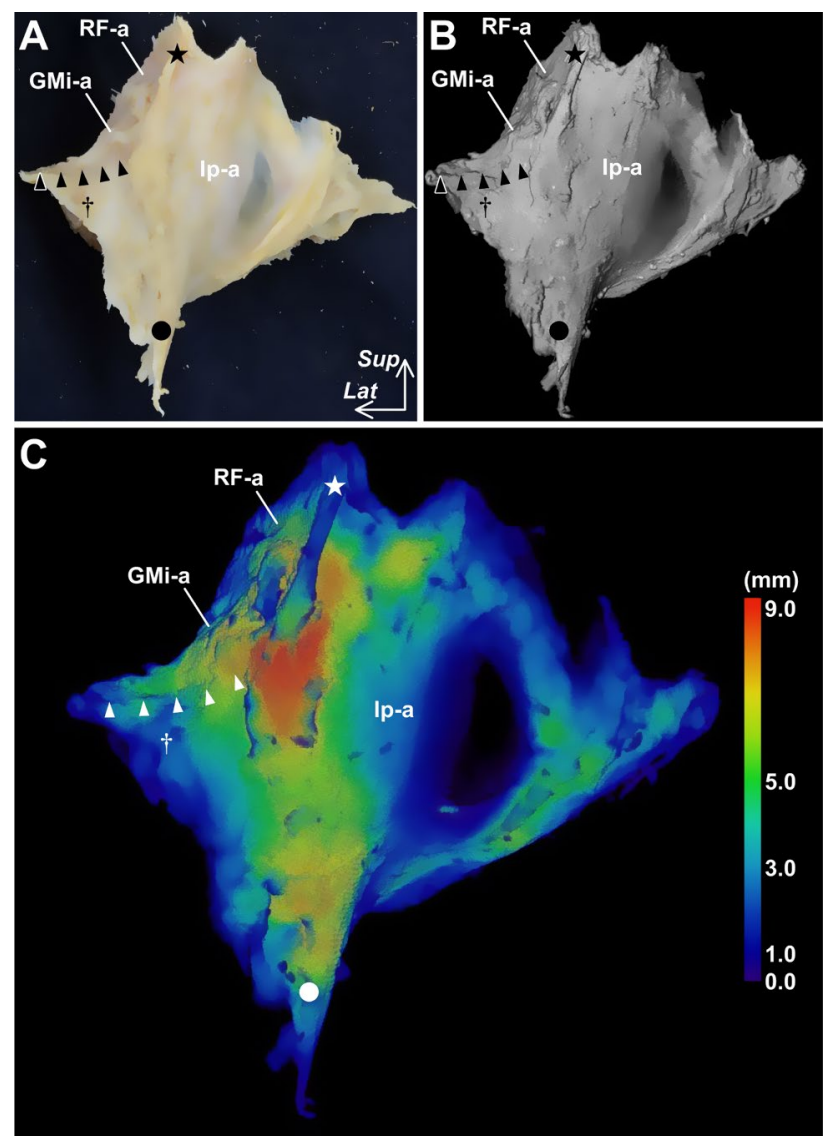

Fig. 5 Outer appearance of the hip joint capsule and its thickness distribution. Anterior aspects of the hip joint capsule. A The hip joint capsule is detached from both the acetabular margin and femur while maintaining the three-dimensional (3D) morphology without cutting its substantial part. B 3D recontraction micro-CT image of A. C Thickness distribution map of $\mathrm{B}$ is visualized after image processing by Bone $\mathrm{J}$, which analyzes the thickness of the structure of interest at a point including more than just bones (Hildebrand and Rüegsegger 1997; Doube et al. 2010). The color bar represents the approximate thickness corresponding to different colors. Arrowheads $=$ cutting line of the connection between the gluteus minimus tendon and the joint capsule; circle $=$ inferomedial end of the intertrochanteric line; dagger, superolateral end of the intertrochanteric line; $I p$ - $a$ deep aponeurosis of the iliopsoas, $G M i-a$ deep aponeuroses of the gluteus minimus, $R F$ - $a$ proximal deep aponeurosis of the rectus femoris, Star inferior area of the anterior inferior iliac spine, Lat lateral and Sup superior (Modified from Tsutsumi et al. 2021)

the muscle. Second, the width of the capsular attachment is wider than previously reported. Finally, the so-called "ligament" has the ability to dynamically coordinate joint stability. These anatomical perspectives provide a better understanding of the hip stabilization mechanism, and a biomechanical study or an in vivo imaging study considering these perspectives is expected in the future.

Acknowledgements This study was supported by JSPS KAKENHI (Grant Number JP 19K18488) and the JA Kyosai Research Institute
(Agricultural Cooperative Insurance Research Institute). We would like to acknowledge and thank the anonymous individuals who generously donated their bodies so that this study could be performed.

Author contributions All the authors contributed to the study conception and design. Material preparation, data collection and analysis were performed by MT. The first draft of the manuscript was written by MT and all the authors commented on previous versions of the manuscript. All the authors read and approved the final manuscript.

Data availability The datasets used and/or analyzed during the current study are available from the corresponding author on reasonable request.

\section{Declarations}

Conflict of interest The authors declare that they have no conflicts of interest.

Open Access This article is licensed under a Creative Commons Attribution 4.0 International License, which permits use, sharing, adaptation, distribution and reproduction in any medium or format, as long as you give appropriate credit to the original author(s) and the source, provide a link to the Creative Commons licence, and indicate if changes were made. The images or other third party material in this article are included in the article's Creative Commons licence, unless indicated otherwise in a credit line to the material. If material is not included in the article's Creative Commons licence and your intended use is not permitted by statutory regulation or exceeds the permitted use, you will need to obtain permission directly from the copyright holder. To view a copy of this licence, visit http://creativecommons.org/licenses/by/4.0/.

\section{References}

Amaha K, Nimura A, Yamaguchi R et al (2019) Anatomic study of the medial side of the ankle base on the joint capsule: an alternative description of the deltoid and spring ligament. J Exp Orthop 6:2

Arai R, Sugaya H, Mochizuki T et al (2008) Subscapularis tendon tear: an anatomic and clinical investigation. Arthroscopy 24:997-1004

Benjamin M, Ralphs JR (1998) Fibrocartilage in tendons and ligaments-an adaptation to compressive load. J Anat 193:481-494

Braus H, Elze C (1954) Bewegungsapparat in Anatomie des Menschen ein Lehrbuch für Studierende und Ärzte. Springer, Berlin

Bunker TD, Esler CN, Leach WJ (1997) Rotator-cuff tear of the hip. J Bone Joint Surg Br 79:618-620

Clark JM, Harryman DT 2nd (1992) Tendons, ligaments, and capsule of the rotator cuff. Gross and microscopic anatomy. J Bone Joint Surg Am 74:713-725

Connell DA, Bass C, Sykes CA et al (2003) Sonographic evaluation of gluteus medius and minimus tendinopathy. Eur Radiol 13:1339-1347

Davies JF, Stiehl JB, Davies JA, Geiger PB (2013) Surgical treatment of hip abductor tendon tears. J Bone Joint Surg Am 95:1420-1425

Doube M, Kłosowski MM, Arganda-Carreras I et al (2010) BoneJ: free and extensible bone image analysis in ImageJ. Bone 47:1076-1079

Fagotti L, Utsunomiya H, Philippon MJ (2020) An anatomic study of the damage to capsular hip stabilizers during subspine decompression using a transverse interportal capsulotomy in hip arthroscopy. Arthroscopy $36: 116-123$ 
Fawcett DW, Bloom W (1986) A textbook of histology. Saunders, Philadelphia

Fry R, Domb B (2010) Labral base refixation in the hip: rationale and technique for an anatomic approach to labral repair. Arthroscopy 26(Suppl):S81-S89

Glick JM, Valone F 3rd, Safran MR (2014) Hip arthroscopy: from the beginning to the future-an innovator's perspective. Knee Surg Sports Traumatol Arthrosc 22:714-721

Gottschalk F, Kourosh S, Leveau B (1989) The functional anatomy of tensor fasciae latae and gluteus medius and minimus. J Anat 166:179-189

Gray H (1858) Anatomy descriptive and surgical. John W. Parker and Son, London

Gray H (1985) Anatomy of the human body. Williams \& Wilkins, Baltimore

Hapa O, Bedi A, Gursan O et al (2013) Anatomic footprint of the direct head of the rectus femoris origin: cadaveric study and clinical series of hips after arthroscopic anterior inferior iliac spine/subspine decompression. Arthroscopy 29:1932-1940

Harris JD, Mccormick FM, Abrams GD et al (2013) Complications and reoperations during and after hip arthroscopy: a systematic review of 92 studies and more than 6,000 patients. Arthroscopy 29:589-595

Hildebrand T, Rüegsegger P (1997) A new method for the modelindependent assessment of thickness in three-dimensional images. J Microsc 185:67-75

Horiuchi S, Nimura A, Tsutsumi M et al (2020) Anatomical relationship between the morphology of the styloid process of the ulna and the attachment of the radioulnar ligaments. J Anat 237:1032-1039

Hoshika S, Nimura A, Yamaguchi R et al (2019) Medial elbow anatomy: a paradigm shift for UCL injury prevention and management. Clin Anat 32:379-389

Kalisvaart MM, Safran MR (2015) Microinstability of the hip-it does exist: etiology, diagnosis and treatment. J Hip Preserv Surg 2:123-135

Kemp JL, Mosler AB, Hart H et al (2020) Improving function in people with hip-related pain: a systematic review and meta-analysis of physiotherapist-led interventions for hip-related pain. Br J Sports Med 54:1382-1394

Kowalczuk M, Bhandari M, Farrokhyar F et al (2013) Complications following hip arthroscopy: a systematic review and meta-analysis. Knee Surg Sports Traumatol Arthrosc 21:1669-1675

Krych AJ, Thompson M, Knutson Z et al (2013) Arthroscopic labral repair versus selective labral debridement in female patients with femoroacetabular impingement: a prospective randomized study. Arthroscopy 29:46-53

Lynch TS, Terry MA, Bedi A, Kelly BT (2013) Hip arthroscopic surgery: patient evaluation, current indications, and outcomes. Am J Sports Med 41:1174-1189

Michal F, Amar E, Atzmon R et al (2020) Subspinal impingement: clinical outcomes of arthroscopic decompression with one year minimum follow up. Knee Surg Sports Traumatol Arthrosc 28:2756-2762

Mochizuki T, Sugaya H, Uomizu M et al (2008) Humeral insertion of the supraspinatus and infraspinatus. New anatomical findings regarding the footprint of the rotator cuff. J Bone Joint Surg Am 90:962-969

Mosler AB, Agricola R, Weir A, Holmich P, Crossley KM (2015) Which factors differentiate athletes with hip/groin pain from those without? A systematic review with meta-analysis. Br J Sports Med 49:810

Myers CA, Register BC, Lertwanich P et al (2011) Role of the acetabular labrum and the iliofemoral ligament in hip stability: an in vitro biplane fluoroscopy study. Am J Sports Med 39(Suppl):85S-91S
Nasu H, Nimura A, Sugiura S et al (2018) An anatomic study on the attachment of the joint capsule to the tibia in the lateral side of the knee. Surg Radiol Anat 40:499-506

Nasu H, Nimura A, Yamaguchi K, Akita K (2020) Morphology of the anterolateral ligament: a complex of fibrous tissues spread to the anterolateral aspect of the knee joint. Anat Sci Int 95:470-477

Neumann DA (2010) Kinesiology of the hip: a focus on muscular actions. J Orthop Sports Phys Ther 40:82-94

Neumann DA (2020) Pelvic girdle, hip, gluteal region and thigh. In: Standring S (ed) Gray's anatomy: the anatomical basis of clinical practice. Elsevier Health Sciences, New York, pp 1352-1394

Nimura A, Kato A, Yamaguchi K et al (2012) The superior capsule of the shoulder joint complements the insertion of the rotator cuff. $\mathrm{J}$ Shoulder Elbow Surg 21:867-872

Nimura A, Fujishiro H, Wakabayashi Y et al (2014) Joint capsule attachment to the extensor carpi radialis brevis origin: an anatomical study with possible implications regarding the etiology of lateral epicondylitis. J Hand Surg Am 39:219-225

Nozaki T, Nimura A, Fujishiro H et al (2015) The anatomic relationship between the morphology of the greater tubercle of the humerus and the insertion of the infraspinatus tendon. J Shoulder Elbow Surg 24:555-560

Pawlina W, Ross MH (2016) Histology: a text and atlas: with correlated cell and molecular biology. Lippincott Williams \& Wilkins, Philadelphia

Pierce TP, Issa K, Kurowicki J et al (2018) Abductor tendon tears of the hip. JBJS Rev 6:e6

Ramos N, Dold A, Youm T (2017) Postoperative considerations following hip arthroscopy. JBJS Rev 5:e3

Safran MR (2019) Microinstability of the hip-gaining acceptance. J Am Acad Orthop Surg 27:12-22

Saka N, Nimura A, Tsutsumi M et al (2021) Anatomic study of fibrous structures attached to the volar ulnar corner of the radius: implications in the volar rim fracture. J Hand Surg Eur. https://doi.org/10.1177/1753193420982192

Sato K, Nimura A, Yamaguchi K, Akita K (2012) Anatomical study of the proximal origin of hamstring muscles. J Orthop Sci $17: 614-618$

Sato T, Nimura A, Yamaguchi R et al (2018) Intramuscular tendon of the adductor pollicis and underlying capsule of the metacarpophalangeal joint: an anatomical study with possible implications for the Stener lesion. J Hand Surg Am 43:682.e1-682.e8

Schafer EA, Thane GD (1894) Quain's elements of anatomy. Longman Green Co, London

Schleip RPD (2012) Fascia: the tensional network of the human body: the science and clinical applications in manual and movement therapy. Churchill Livingstone/Elsevier, New York

Segal NA, Felson DT, Torner JC et al (2007) Greater trochanteric pain syndrome: epidemiology and associated factors. Arch Phys Med Rehabil 88:988-992

Semciw AI, Pizzari T, Murley GS, Green RA (2013) Gluteus medius: an intramuscular EMG investigation of anterior, middle and posterior segments during gait. J Electromyogr Kinesiol $23: 858-864$

Shimura H, Nimura A, Nasu H et al (2016) Joint capsule attachment to the coronoid process of the ulna: an anatomic study with implications regarding the type 1 fractures of the coronoid process of the O’Driscoll classification. J Shoulder Elbow Surg 25:1517-1522

Tamaki T, Nimura A, Oinuma K et al (2014) An anatomic study of the impressions on the greater trochanter: bony geometry indicates the alignment of the short external rotator muscles. J Arthroplasty 29:2473-2477

Tamaki Y, Goto T, Wada K et al (2020) Anatomic evaluation of the insertional footprints of the iliofemoral and ischiofemoral ligaments: a cadaveric study. BMC Musculoskelet Disord 21:828 
Tano A, Nimura A, Tsutsumi M et al (2021) Anatomical study of the interosseous ligament of the tibiofibular syndesmosis: an analysis of osseous morphology and attaching interposing structures. J Bone Joint Surg Am 103:905-912

Telleria JJ, Lindsey DP, Giori NJ, Safran MR (2014) A quantitative assessment of the insertional footprints of the hip joint capsular ligaments and their spanning fibers for reconstruction. Clin Anat 27:489-497

Tsutsumi M, Nimura A, Akita K (2019a) The gluteus medius tendon and its insertion sites: an anatomical study with possible implications for gluteus medius tears. J Bone Joint Surg Am 101:177-184

Tsutsumi M, Nimura A, Honda E et al (2019b) An anatomical study of the anterosuperior capsular attachment site on the acetabulum. J Bone Joint Surg Am 101:1554-1562

Tsutsumi M, Nimura A, Akita K (2020) New insight into the iliofemoral ligament based on the anatomical study of the hip joint capsule. J Anat 236:946-953

Tsutsumi N, Nimura A, Utsunomiya H, Akita K (2021) Dynamic changes of the joint capsule in relation to the zona orbicularis: An anatomical study with possible implications for hip stability mechanism. Clin Anat 34(8):1157-1164. https://doi.org/10.1002/ ca. 23767
Wagner FV, Negrão JR, Campos J et al (2012) Capsular ligaments of the hip: anatomic, histologic, and positional study in cadaveric specimens with MR arthrography. Radiology 263:189-198

Walters BL, Cooper JH, Rodriguez JA (2014) New findings in hip capsular anatomy: dimensions of capsular thickness and pericapsular contributions. Arthroscopy 30:1235-1245

Wolff J (1892) Das Gesetz der Transformation der Knochen, Berlin, A. Hirchwild. Springer, Berlin

Yeung M, Memon M, Simunovic N et al (2016) Gross instability after hip arthroscopy: an analysis of case reports evaluating surgical and patient factors. Arthroscopy 32:1196-1204.e1

Zhu MF, Musson DS, Cornish J et al (2020) Hip abductor tendon tears: where are we now? Hip Int 30:500-512

Publisher's Note Springer Nature remains neutral with regard to jurisdictional claims in published maps and institutional affiliations. 